\title{
Homes Without Quality
}

\author{
BRITTANY UTTING \\ University of Michigan
}

The developer's catalog of homes is the most persuasive author of the suburban landscape. This database of houses-a combinatorics of style and typology-constitutes a logistical regime of wealth distribution, territorial acquisition, and aesthetic management. The catalog's listings of architectural style and model number establishes a strategy that leverages the iconography of the home to clad otherwise identical floor plans. The question then arises: what becomes of the standardized, market-generated plan in a post-digital city? These domestic objects-generic in disposition and typified by the city-are an alternative to the positivisms of parametric urbanism, proposing architecture as an open framework, one through which we can construct new models of collective life, leisure, and labor.

Each of these model homes is an abstraction of the programs of domestic life: where to eat, sleep, bathe, work, and play. By reducing the home to a series of rooms specified by furnishings and fixtures, the generic diagram allows us to decouple the home from the flows of wealth, debt, efficiency analytics, and market-driven data that manage suburban inhabitation, instead serving subjectivities not currently accommodated. This project constitutes a new formal paradigm to counter parametric flows of suburban deployment and compliance, co-opting the ethos of mass production and typological reconfiguration to produce new models of cohabitation, consumption, and production.

Employing deadpan combinatorics, material distribution systems, and organizational logistics, this alternative set of model homes appropriates the same paradigms of abstraction employed by Levittown and the Sears Catalog to reconstruct the 'useless machine' of development. The appropriation of the catalog's indifferent mechanism of house deployment leverages the open frame of the domestic generic to produce a new suburban landscape. Tying political embodiment to architectural form, these new permutations belong to a more radical catalog of domestic models - characterized by minimalisms and excesses, exotic layouts and redundancies. The positioning of each model in its lot results in a more exotic urban space, one that resists the homogeneity expected from typical suburban developments. Each type becomes a proxy for new patterns of occupancy, use, and commerce within and around the home. The resulting suburban terrain creates new confrontations, new courtyards and clearings, more obstructions but also more routes and itineraries.
Characterized by typological excess and topological diversity, this catalog constitutes the protocols for a new suburb, an alternative fabric in which to inscribe more varied forms of public and private life. These unfamiliar re-arrangements of domestic objects suggest new forms of collectivity and kinship within the monumentalized suburban field. They make space for habits negotiation and cooperation, inevitably generating new hierarchies of power and territory; they enact alternative patterns of use, ownership, and occupation to challenge conditions of access, stewardship, and consensus. Rather than participating in the dominant paradigm of suburban development-the mass-customization of signature homes-there is instead a new litany of domestic models. This catalog of Homes Without Quality appropriates the generic framework of type to resist the market of lifestyleas-image, enacting architectural form as political form. 


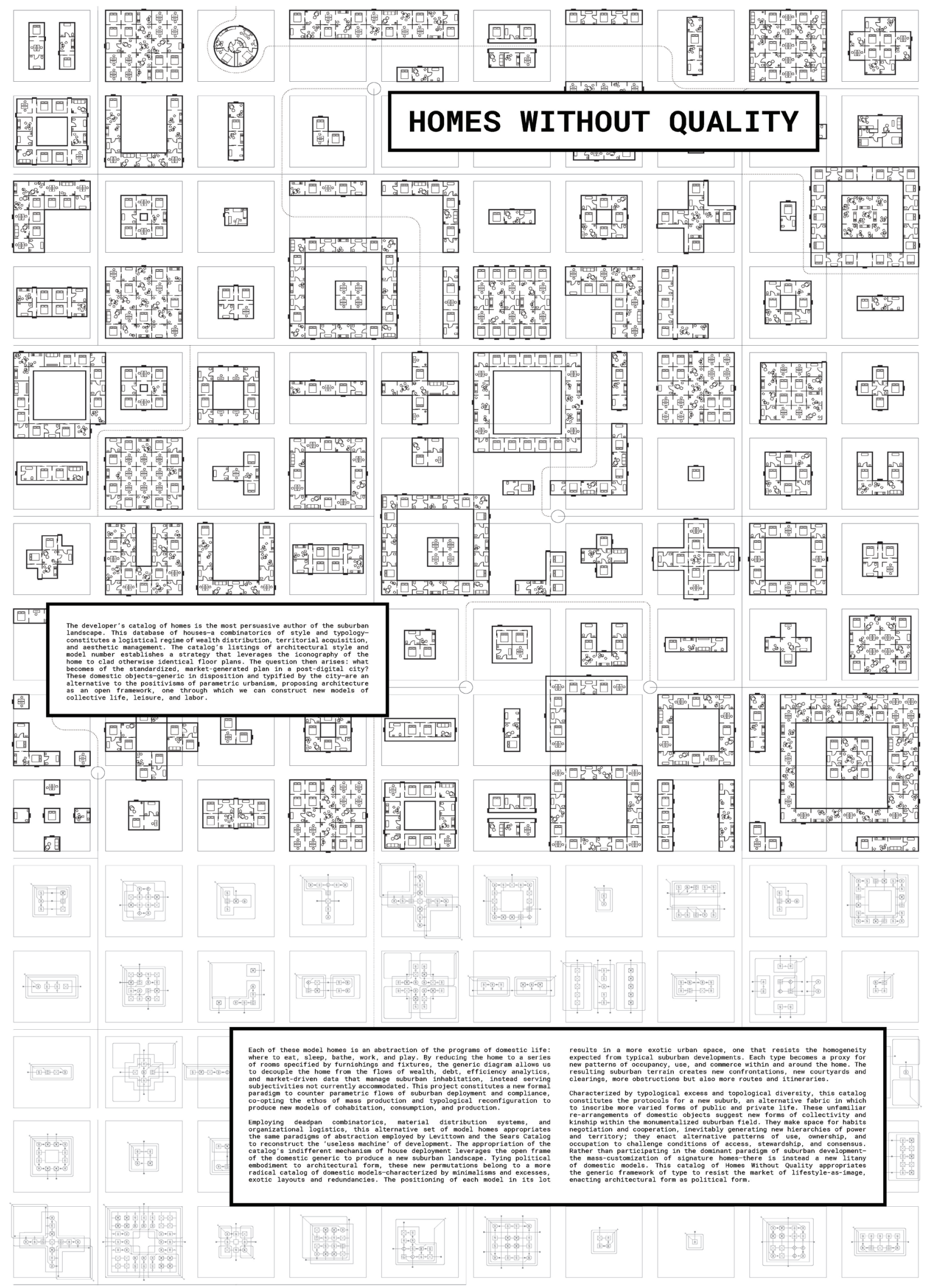

